\title{
Osteosarcoma of the Proximal Femur Mimicking Septic Arthritis of the Hip A diagnostic dilemma
}

"Salil Umrani and Yaqoub Al-Mufargi

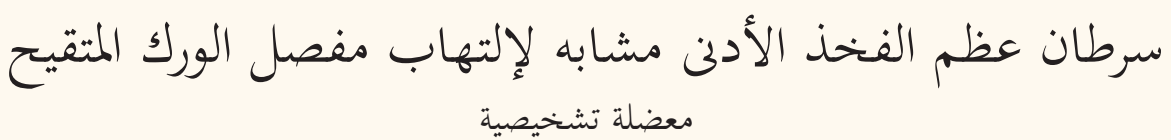

سليل عمراني و يعقوب المفرجي

ABSTRACT: Septic arthritis is a surgical emergency requiring prompt drainage of the accumulation of pus in the joint. We report an eight-year-old boy who was referred to the Armed Forces Hospital, Salalah, Oman, in 2016 with limping and pain in his right hip along with an abduction deformity. He was initially diagnosed with synovitis and underwent conservative management. However, two weeks later, he presented to another centre due to persistent pain. Magnetic resonance imaging was suggestive of septic arthritis or osteomyelitis. Eight weeks later, the patient presented again to the Armed Forces Hospital. An arthrotomy was performed, during which an amount of what appeared to be frank pus was drained; however, cultures of the intraoperative samples returned no growth. A histopathological analysis revealed an osteosarcoma. The patient was subsequently referred elsewhere and underwent neoadjuvant chemotherapy and limb salvage surgery. Several months later, he relapsed and developed extensive pulmonary metastasis and died as a result of progressive deterioration.

Keywords: Osteosarcoma; Hip Joint; Septic Arthritis; Osteomyelitis; Differential Diagnosis; Case Report; Oman.

الملخص: يعتبر إلتهاب المفاصل التقيحي من حالات الطوارئ الحرجة التي تتطلب تدخلاً جراحياً سريعاً لتصريف القيح المتراكم في

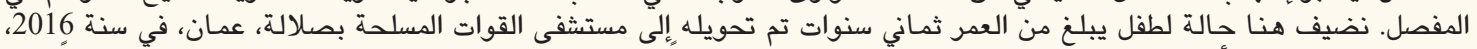

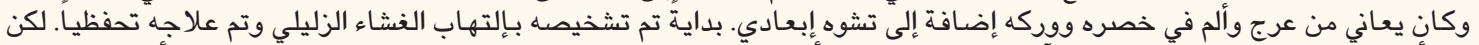

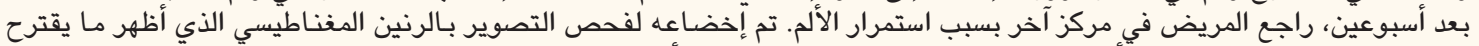

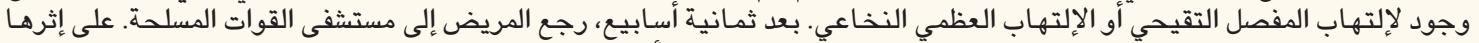

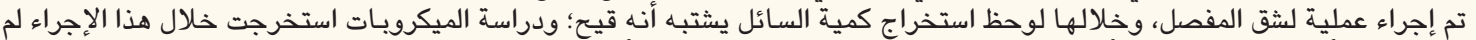

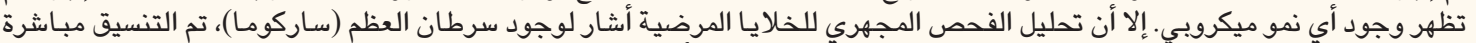

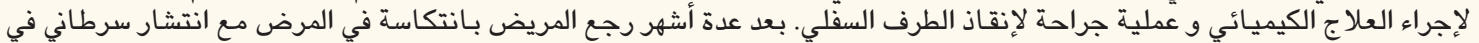
الرئة ومات نتيجة تدهور في حالته. الكلمات المفتاحية: سرطان عظمي؛ مفصل الورك؛ إلتهاب المفاصل التقيحي؛ إلتهاب العظمي النخاعي؛ التشخيص التفريقي؛ تقرير حالة؛ عمان.

$\mathrm{O}$ STEOSARCOMAS ARE CHARACTERISED BY THE production of osteoid material by malignant cells. ${ }^{1}$ Primary osteosarcomas can be classified into conventional, low-grade intramedullary, parosteal, periosteal, high-grade surface, telangiectatic and smallcell types and are the second most common type of primary bone malignancy, with an incidence of 1-3 cases per million people per year. Onset can occur at any age and patients usually present with pain which improves initially and then worsens, particularly at night. ${ }^{1}$ The differential diagnosis of an osteosarcoma includes benign bone tumours, infections and other malignant disorders. ${ }^{2}$

Among these differential diagnoses, septic arthritis in particular can present very similarly to an osteosarcoma. $^{2,3}$ This condition involves the accumulation of pus in a joint and can potentially result in severe sequelae such as the destruction or occlusion of the epiphysis; as such, urgent diagnosis and drainage of the accumulated pus is required. ${ }^{3}$ This report describes an osteosarcoma case which initially presented with features of septic arthritis and highlights various aspects of these two conditions to allow for prompt differentiation and treatment.

\section{Case Report}

An eight-year-old boy was referred to the Orthopaedic Clinic of the Armed Forces Hospital, Salalah, Oman, in 2016 with pain in his right hip and noticeable limping following a trivial fall. The pain was constant, abating only intermittently with analgaesics. There was diffuse tenderness in the right hip with limited flexion (30-90 degrees), fixed abduction (30 degrees), restricted rotation, 


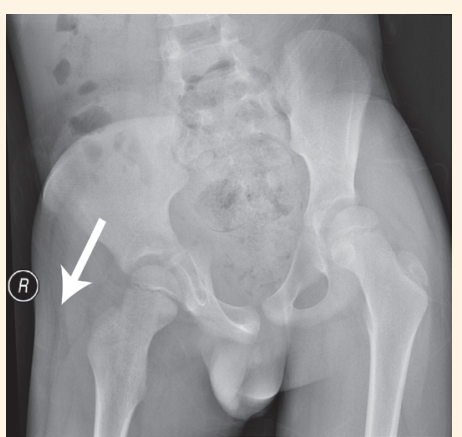

Figure 1: X-ray of the pelvis of an eight-year-old boy with right hip pain at presentation showing pelvic obliquity and an abduction deformity in the right hip (arrow).

apparent leg lengthening and pelvic obliquity. His white blood cell count (WBC), erythrocyte sedimentation rate (ESR) and C-reactive protein (CRP) levels $\left(5.43 \times 10^{9} / \mathrm{L}\right.$, $6 \mathrm{~mm} /$ hour and $1.04 \mathrm{mg} / \mathrm{L}$, respectively) were within normal ranges $\left(4.8-10.8 \times 10^{9} / \mathrm{L}, 0-20 \mathrm{~mm} /\right.$ hour and 0-5 mg/L, respectively). An X-ray confirmed the pelvic obliquity and an abduction deformity in the right hip [Figure 1].

An initial working diagnosis of synovitis was made and conservative management was advised. However, the patient was subsequently lost to follow up. Two weeks later, he was admitted elsewhere and underwent magnetic resonance imaging (MRI); this revealed joint effusion in the right hip with marrow signal changes [Figure 2]. He was again treated conservatively for synovitis and subsequently discharged. As the pain persisted, the patient presented to yet another centre after one month, at which point he was suspected to have histiocytosis or infective synovitis. However, a repeated blood work-up indicated that his WBC, ESR and CRP levels were normal. Nevertheless, MRI revealed moderate effusion in the right hip with thickening of the femur neck and bone marrow oedema, suggestive of septic arthritis of the right hip and osteomyelitis of the proximal femur [Figure 3]. The patient was discharged without treatment.

Eight weeks later, the patient presented once again to the Armed Forces Hospital with persistent complaints of pain and limping. There was no history of fever, dysuria or involvement of any joints other than the hip during this period. His WBC, ESR and CRP levels were still normal $\left(5.13 \times 10^{9} / \mathrm{L}, 20 \mathrm{~mm} /\right.$ hour and $1.66 \mathrm{mg} / \mathrm{L}$, respectively). Blood and urine cultures revealed no bacterial growth. However, an X-ray revealed periarticular osteopaenia and a possible lytic lesion in the proximal femur of the right hip [Figure 4]. The patient was referred to the Paediatric Orthopaedic Unit of the Armed Forces Hospital in Muscat, Oman. At this time, repeat investigations indicated raised ESR and CRP levels $(32.97 \mathrm{mg} / \mathrm{L}$ and $51 \mathrm{~mm}$ /hour, respectively).

An arthrotomy was immediately performed, revealing what appeared to be thick pus originating from the hip joint and the neck of the femur along with unhealthy tissue around the latter. Following thorough curettage and copious saline lavage (approximately $6 \mathrm{~L}$ ), samples of the pus-like material were sent for Gram staining and cultures for routine bacteria as well as acid-fast bacilli and fungi. A histopathological examination was also requested. In the meantime, antibiotic therapy was initiated; however, although the patient appeared to improve clinically in the initial postoperative period, he subsequently reverted to his preoperative status. While his WBC remained normal (range: $5.8-7.4 \times 10^{9} / \mathrm{L}$ ) and his CRP levels gradually returned to normal (from $12.2 \mathrm{mg} / \mathrm{L}$ to $3.1 \mathrm{mg} / \mathrm{L}$ ), his ESR remained elevated (range: $45-55 \mathrm{~mm} /$ hour) throughout the postoperative period.

Subsequently, the biopsy results revealed that none of the intraoperative cultures had resulted in growth. The histopathological analysis confirmed a diagnosis of an osteosarcoma (chondroblastic variant). However, postoperative $\mathrm{X}$-rays revealed the same findings as those performed preoperatively and a postoperative chest $\mathrm{X}$-ray was normal. The patient was referred to a paediatric oncology unit in Muscat, where a staging work-up revealed single bilateral pulmonary metastases. Neoadjuvant chemotherapy was initiated as per institutional protocols. A few months later, following completion of the neoadjuvant chemotherapy regimen, he underwent limb salvage surgery in India; at this time, a histological analysis revealed 50-60\% tumour response to treat-
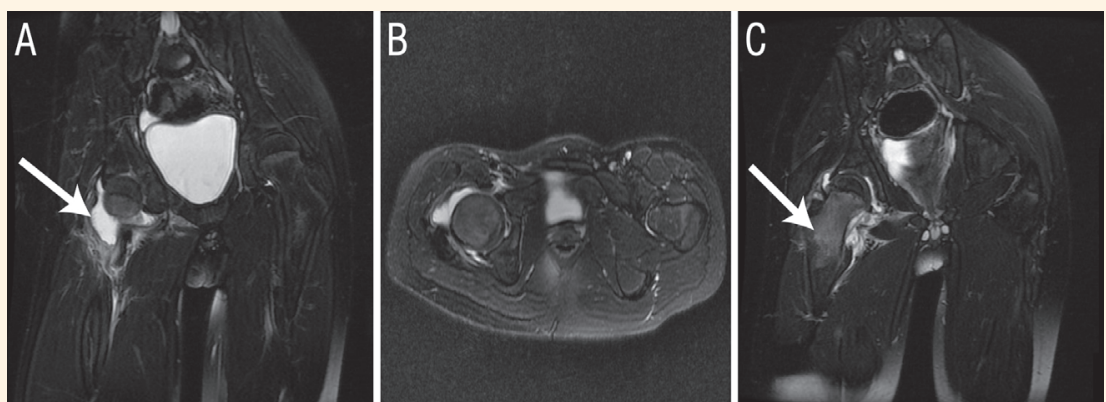

Figure 2: Magnetic resonance imaging of an eight-year-old boy with persistent right hip pain showing joint effusion in the right hip with marrow signal changes (arrows). The patient was provisionally diagnosed with synovitis. 

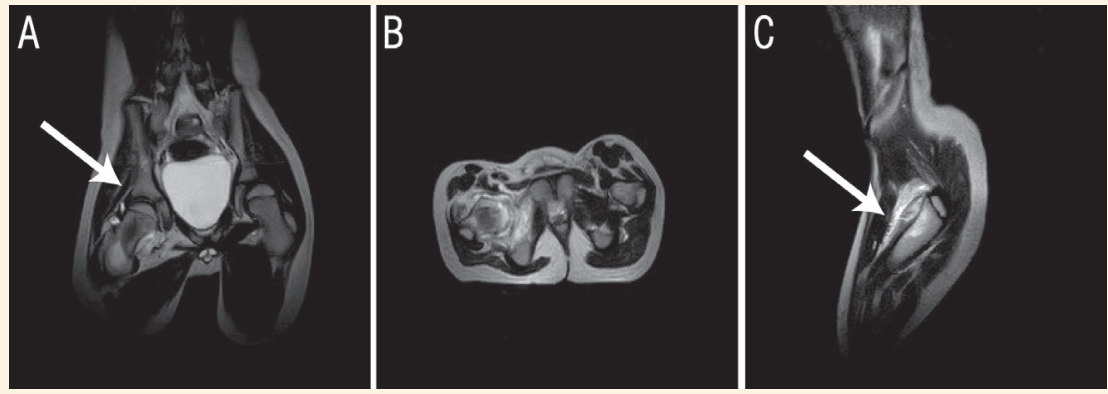

Figure 3: Magnetic resonance imaging of an eight-year-old boy with persistent right hip pain showing moderate effusion in the right hip with thickening of the femur neck and bone marrow oedema (arrows).

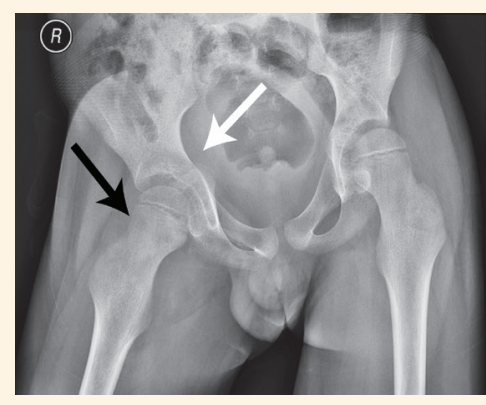

Figure 4: X-ray of the pelvis of an eight-year-old boy with persistent right hip pain after eight weeks showing periarticular osteopaenia (white arrow) and a possible lytic lesion in the proximal femur of the right hip (black arrow).

ment. Adjuvant chemotherapy was resumed after the surgery; however, the patient relapsed and developed multiple bilateral pulmonary metastases and septic shock. Unfortunately, although he recovered from the shock with intensive treatment, the patient died a few months later as his condition progressively deteriorated.

\section{Discussion}

In the current case, the patient was initially diagnosed with synovitis, followed by septic arthritis and osteomyelitis, before the correct diagnosis of an osteosarcoma was made based on the histopathological findings. Transient synovitis is a common self-limiting condition which improves rapidly with conservative treatment. ${ }^{4}$ In the present case, this was eventually ruled out in view of the patient's prolonged illness. Children with acute septic arthritis of the lower limbs usually present with fever, pain and limping; moreover, they often refuse to put weight on the affected leg or hold the affected joint in a position of comfort. ${ }^{5}$

Although the current patient presented initially with pain and refused to bear weight on the affected limb, he did not have fever. Furthermore, blood cultures as well as arthrotomy samples yielded no growth of nongonococcal bacteria or evidence of polymorphonuclear predominance. ${ }^{6}$ Unfortunately, recent advances in the early detection of septic arthritis-including synovial procalcitonin estimations, combined leukocyte esterase/ glucose strip tests, $\alpha$-defensin synovial fluid measurements and next-generation DNA sequencing-were unavailable at the time of presentation. ${ }^{7-10}$

Kocher et al. proposed certain criteria to help diagnose septic arthritis, with at least three of the following four conditions indicating a 93-99\% risk of septic arthritis: (1) lack of weight-bearing on the affected side; (2) ESR of $>40 \mathrm{~mm} /$ hour; (3) fever of $>38.5^{\circ} \mathrm{C}$; and (4) WBC of $>12,000$ cells $/ \mathrm{mm}^{3} .11,12$ The risk falls to $40 \%$ if only two of the criteria are met, dwindling to $3 \%$ if only one criterion is met. ${ }^{11,12}$ Kang et al. similarly confirmed that an inability to bear weight, fever and a raised WBC are diagnostic of septic arthritis, with a sensitivity of $>98 \% .{ }^{13}$ In the current case, the patient met only the first criterion in that he could not bear weight on the affected side. In addition, repeated $\mathrm{X}$-rays did not demonstrate any sequelae of septic arthritis, such as destruction or occlusion of the epiphysis. ${ }^{3}$ As a result, there was no suspicion of septic arthritis at initial presentation. However, following an increase in ESR and CRP levels, an arthrotomy was immediately performed.

On radiology, septic arthritis has typical imaging features, including periarticular soft tissue swelling, effacement with blurring of the periarticular fat planes, effusion and periarticular osteopaenia in the early stages, followed by narrowing and destruction of the joint space with premature osteoarthritis and shortening of the limb in the later stages. ${ }^{14}$ In contrast, an osteosarcoma appears as an aggressive metaphyseal blastic or lytic lesion, with areas of bone production and bone destruction; in addition, a permeative ill-defined soft tissue mass may be visible. ${ }^{1}$ The periosteal reaction forms either Codman's triangle, sunburst or 'hair-on-end' patterns. In the present case, plain X-rays showed consistent evidence of persistent periarticular osteopaenia and a lytic lesion in the proximal femur over a three-month period, even after the arthrotomy was performed. 
Ultrasonography may also be helpful to diagnose septic arthritis and guide aspiration. ${ }^{14}$ The fluid in the joint may either be hypoechoic and clearly separated from or hyperechoic and adjacent to the joint capsule. Furthermore, in children, the capsule is $>5 \mathrm{~mm}$ away from the femoral cortex and/or $>2 \mathrm{~mm}$ thicker than that of the normal side. ${ }^{14}$ Power Doppler sonography may also show increased capsular vascularity. However, in the current case, ultrasonography was not requested as the patient had already undergone repeated MRI scans and X-rays.

Most osteosarcomas begin in an intramedullary location but may pierce through the cortex to form a soft tissue mass. ${ }^{1}$ Histologically, they can be osteoblastic, fibroblastic or chondroblastic. The diagnosis is usually delayed since common musculoskeletal conditions need to be excluded first. Pan et al. reported an average delay of approximately 17 weeks from the onset of symptoms to diagnosis. ${ }^{15}$ Both patient- and physicianrelated factors may be responsible for this delay; the latter include a failure to obtain X-rays at presentation and/or after symptoms persist or worsen. ${ }^{1}$ In the present case, X-rays were taken at both initial and follow-up visits; however, these did not demonstrate evidence of any lumps, masses or significant changes indicative of an osteosarcoma. Unfortunately, although the correct diagnosis was made in under 17 weeks, it is likely that the patient would have been diagnosed much earlier and possibly had a better prognosis if he had been followed-up regularly at a single centre.

The long-term survival rate reported for highgrade osteosarcomas without metastasis at presentation is $60-70 \%$, rising to $90 \%$ for low-grade lesions; however, this decreases rapidly to $20 \%$ if there are detectable pulmonary metastases at diagnosis. ${ }^{1}$ Although the current patient had a clear chest X-ray when the diagnosis was made, the staging work-up revealed bilateral single pulmonary metastatic nodules. Most patients with high-grade osteosarcomas have non-detectable micrometastases at presentation. ${ }^{1}$ Even with neoadjuvant or adjuvant chemotherapy, approximately $50 \%$ of patients relapse after initial treatment, as seen in the current case. $^{1}$

\section{Conclusion}

This case report describes a patient who was initially diagnosed with synovitis, followed by septic arthritis and osteomyelitis, before the correct diagnosis of an osteosarcoma was made based on histopathological findings. Infection is a known differential diagnosis of malignancy but must be identified quickly and treated promptly. Physicians should be aware that osteosarcomas may present with features suggestive of septic arthritis and osteomyelitis.

\section{References}

1. Heck RK Jr. Malignant tumors of bone. In: Canale ST, Beaty JH, Eds. Campbell's Operative Orthopaedics, 12th ed. Philadelphia, Pennsylvania, USA: Elsevier Mosby, 2008. Pp. 909-14.

2. ESMO/European Sarcoma Network Working Group. Bone sarcomas: ESMO clinical practice guidelines for diagnosis, treatment and follow-up. Ann Oncol 2014; 25:iii113-23. https://doi. org/10.1093/annonc/mdu256.

3. Shaw BA, Kasser JR. Acute septic arthritis in infancy and childhood. Clin Orthop Relat Res 1990; 257:212-25.

4. Luhmann SJ, Jones A, Schootman M, Gordon JE, Schoenecker PL, Luhmann JD. Differentiation between septic arthritis and transient synovitis of the hip in children with clinical prediction algorithms. J Bone Joint Surg Am 2004; 86-A:956-62.

5. Gutierrez K. Bone and joint infections in children. Pediatr Clin North Am 2005; 52:779-94. https://doi.org/10.1016/j. pcl.2005.02.005.

6. Goldenberg DL. Septic arthritis. Lancet 1998; 351:197-202. https://doi.org/10.1016/S0140-6736(97)09522-6.

7. Wang C, Zhong DA, Liao Q, Kong L, Liu A, Xiao H. Procalcitonin levels in fresh serum and fresh synovial fluid for the differential diagnosis of knee septic arthritis from rheumatoid arthritis, osteoarthritis and gouty arthritis. Exp Ther Med 2014; 8:1075-80. https://doi.org/10.3892/etm.2014.1870.

8. Omar M, Ettinger M, Reichling M, Petri M, Lichtinghagen R, Guenther D, et al. Preliminary results of a new test for rapid diagnosis of septic arthritis with use of leukocyte esterase and glucose reagent strips. J Bone Joint Surg Am 2014; 96:2032-7. https://doi.org/10.2106/JBJS.N.00173.

9. Deirmengian C, Kardos K, Kilmartin P, Cameron A, Schiller K, Parvizi J. Combined measurement of synovial fluid $\alpha$-defensin and C-reactive protein levels: Highly accurate for diagnosing periprosthetic joint infection. J Bone Joint Surg Am 2014; 96:1439-45. https://doi.org/10.2106/JBJS.M.01316.

10. Tarabichi M, Shohat N, Goswami K, Alvand A, Silibovsky R, Belden K, et al. Diagnosis of periprosthetic joint infection: The potential of next-generation sequencing. J Bone Joint Surg Am 2018; 100:147-54. https://doi.org/10.2106/JBJS.17.00434.

11. Kocher MS, Zurakowski D, Kasser JR. Differentiating between septic arthritis and transient synovitis of the hip in children: An evidence-based clinical prediction algorithm. J Bone Joint Surg Am 1999; 81:1662-70.

12. Kocher MS, Mandiga R, Zurakowski D, Barnewolt C, Kasser JR. Validation of a clinical prediction rule for the differentiation between septic arthritis and transient synovitis of the hip in children. J Bone Joint Surg Am 2004; 86-A:1629-35.

13. Kang SN, Sanghera T, Mangwani J, Paterson JM, Ramchandran M. The management of septic arthritis in children: Systematic review of the English language literature. J Bone Joint Surg Br 2009; 91:1127-33. https://doi.org/10.1302/0301-620X.9 1B9.22530.

14. Chau CL, Griffith JF. Musculoskeletal infections: Ultrasound appearances. Clin Radiol 2005; 60:149-59. https://doi.org/10.10 16/j.crad.2004.02.005.

15. Pan KL, Chan WH, Chia YY. Initial symptoms and delayed diagnosis of osteosarcoma around the knee joint. J Orthop Surg (Hong Kong) 2010; 18:55-7. https://doi.org/10.1177/2309 49901001800112 